\title{
Fatores associados a atraso no diagnóstico da síndrome de Turner
}

\author{
Variables associated with diagnostic delay in Turner syndrome
}

Jamil Miguel Neto ${ }^{1}$, Sofia Helena V. L. Marini ${ }^{2}$ Antonia Paula M. Faria ${ }^{3}$, Gil Guerra Júnior ${ }^{4}$, Andréa Trevas M. Guerra

\section{RESUMO}

Objetivo: Investigar as possíveis razões do atraso no diagnóstico da síndrome de Turner (ST), ou seja, aquele realizado após a idade em que se pode estabelecer o atraso puberal.

Métodos: Estudo transversal com obtenção de dados dos prontuários de 29 pacientes com ST diagnosticadas com mais de dois anos, entre 2004 e 2007. Foram comparados antecedentes pessoais e familiares e dados de exame físico das pacientes diagnosticadas com menos de 13 anos (limite a partir do qual se pode caracterizar atraso puberal em meninas) com os daquelas diagnosticadas após os 13 anos por meio dos testes $t$ de Student e exato de Fisher.

Resultados: Não houve diferenças significativas quanto à estatura materna e da própria paciente, história de afecções associadas (consideradas individualmente), escolaridade dos pais, recorrência familiar de baixa estatura, presença de cada sinal dismórfico isoladamente e total de sinais observados. Os dois grupos diferiram quanto à presença de ao menos uma afecção sugestiva dessa síndrome (associada ao diagnóstico mais precoce) e ao número de irmãos (maior no diagnóstico tardio e associado à menor escolaridade materna).

Conclusões: $\mathrm{O}$ diagnóstico precoce relacionou-se mais à presença de alguma das afecções associadas à ST (possivelmente determinando-se encaminhamento a serviços de maior complexidade) do que a sinais dismórficos. Há indicações de que déficit de crescimento menos evidente, dificuldade dos médicos em reconhecer anomalias sugestivas dessa síndrome

Instituição: Faculdade de Ciências Médicas da Universidade Estadual de Campinas (Unicamp), Campinas, SP, Brasil

${ }^{1}$ Aluno do Curso de Graduação em Medicina da Faculdade de Ciências Médica da Unicamp, Campinas, SP, Brasil

${ }^{2}$ Doutora em Saúde da Criança e do Adolescente pela Faculdade de Ciências Médicas da Unicamp; Professora do Departamento de Pediatria da Faculdade de Ciências Médicas da Unicamp, Campinas, SP, Brasil 3Livre-Docente em Genética Clínica pela Faculdade de Ciências Médicas da Unicamp; Professora Associada do Departamento de Genética Médica da Faculdade de Ciências Médicas da Unicamp, Campinas, SP, Brasil ${ }^{4}$ Livre-Docente em Pediatria pela Faculdade de Ciências Médicas da Unicamp; Professor Associado do Departamento de Pediatria da Faculdade de Ciências Médicas da Unicamp, Campinas, SP, Brasil

${ }^{5}$ Livre-Docente em Genética Clínica pela Faculdade de Ciências Médicas da Unicamp; Professora Titular do Departamento de Genética Médica da Faculdade de Ciências Médicas da Unicamp, Campinas, SP, Brasil e determinantes socioeconômicos contribuam para o atraso no diagnóstico. É necessário enfatizar na formação pediátrica o reconhecimento do espectro clínico dessa síndrome e ampliar os serviços públicos de genética.

Palavras-chave: síndrome de Turner; aberrações cromossômicas; diagnóstico precoce; anormalidades congênitas.

\section{ABSTRACT}

Objective: To investigate the possible reasons for diagnostic delay in Turner syndrome (TS), i.e., a diagnosis made after the age when pubertal delay may be established.

Methods: Cross-sectional study with data obtained from the records of 29 TS patients aged more than two years who were diagnosed between 2004 and 2007. Data on personal and family history and physical examination from patients diagnosed before 13 years old (age limit from which pubertal delay may be characterized in girls) were compared to those of girls diagnosed after 13 years by Fisher exact test and Student's t-test.

Results: No significant differences were noted regarding mothers' and patients' stature, personal history of TS-associated diseases (considered individually), parental schooling, familial recurrence of short stature, presence of each dysmorphic feature considered separately, and total number of dysmorphic features. The two groups differed regarding the presence of at least one TS-associated disease

Endereço para correspondência

Andréa Trevas M. Guerra

Caixa Postal 6111

CEP13083-970 - Campinas/SP

E-mail: atmg@uol.com.br

Fonte financiadora: Programa Institucional de Bolsas de Iniciação Científica/ Conselho Nacional de Desenvolvimento Científico e Tecnológico (Pibic/ CNPq).

Conflito de interesse: nada a declarar

Recebido em: 3/2/2010

Aprovado em: 8/6/2010 
(which was associated to early diagnosis) and number of siblings (which was higher among patients with delayed diagnosis and associated with lower maternal schooling).

Conclusions: Early diagnosis was more associated with the presence of a TS-associated disease (which may have required referral to secondary or tertiary health care services) than with the presence of dysmorphic signs. The results indicate that less evident growth deficit, physicians' inability to recognize abnormalities associated with TS and socioeconomic aspects may contribute to diagnostic delay. Pediatric training should emphasize recognition of the clinical spectrum of TS and public genetic services should be expanded.

Key-words: Turner syndrome; chromosome aberrations; early diagnosis; congenital abnormalities.

\section{Introdução}

A síndrome de Turner (ST) é determinada pela presença de um cromossomo $\mathrm{X}$ e ausência total ou parcial do segundo cromossomo sexual, $\mathrm{X}$ ou $\mathrm{Y}$, o que afeta a expressão ou regulação de genes neles localizados. No cromossomo X, a região crítica para o surgimento dos sinais da ST está no braço curto (Xp11.2 - p22.1) ${ }^{(1)}$. Incide em cerca de 1:2.500 nascidos vivos de sexo feminino ${ }^{(2)}$; porém, entre meninas com baixa estatura proporcionada e bom desenvolvimento neuropsicomotor atendidas em serviço de Pediatria de hospital universitário, sua frequência sobe para $12,5 \% \%^{(3)}$.

Os principais sinais clínicos são baixa estatura e disgenesia gonadal. Podem ainda ser observados vários dismorfismos, como implantação baixa de cabelos na nuca; estrabismo; ptose palpebral; palato ogival; micrognatia; pescoço curto; pescoço alado; linfedema no dorso de mãos e/ou pés; encurtamento de metacarpianos; deformidade de Madelung; cubitus valgus; genu valgum; escoliose e múltiplos nevos pigmentados; anomalias congênitas, particularmente cardiovasculares e renais; afecções adquiridas, como doenças tireoidianas, deficiência auditiva, hipertensão, osteoporose e obesidade e também problemas psicossociais ${ }^{(4-7)}$. É grande a variabilidade fenotípica, desde pacientes com quadro dismórfico exuberante até aqueles quase indistinguíveis da população geral.

A relevância do diagnóstico precoce da ST está em permitir também o diagnóstico precoce de anomalias congênitas e adquiridas e a instituição das medidas terapêuticas adequadas; permitir detectar casos com cromossomo Y no cariótipo, nos quais o surgimento de neoplasias gonadais pode ser evitado com gonadectomia profilática; além de permitir que tratamentos de promoção do crescimento e reposição de hormônios sexuais sejam feitos na idade adequada, evitandose danos adicionais à saúde dos pacientes ${ }^{(5)}$.

No entanto, em muitos casos, o diagnóstico só é feito após o início da adolescência, quando caracteriza-se o atraso puberal - em nosso meio, a média de idade ao diagnóstico é de 12 anos, com desvio padrão de 7,1 anos ${ }^{(8)}$. É possível que o atraso no diagnóstico de algumas pacientes se deva à variabilidade fenotípica, de modo que aquelas sem dismorfismos evidentes sejam diagnosticadas mais tarde. Além disso, a experiência clínica mostra que muitas mães, ao buscarem atendimento médico para suas filhas com queixa de deficiência de crescimento, atribuem esta à história familiar de baixa estatura, principalmente materna.

Por outro lado, o achado de déficit de crescimento mais acentuado, a presença de afecções frequentemente associadas à ST, como doença tireoideana autoimune e anomalias cardíacas, renais e/ou de vias urinárias, e a comparação com o crescimento de irmãos e irmãs normais podem chamar a atenção do pediatra para o caráter patológico da deficiência de crescimento. Além disso, a maior escolaridade dos pais pode ainda favorecer a procura por atendimento médico.

À vista da importância do diagnóstico precoce da ST e das diversas hipóteses para explicar a ampla variação na idade ao diagnóstico dessas pacientes, este trabalho teve por objetivo investigar as possíveis razões do atraso no diagnóstico, aqui considerado como aquele realizado após a idade em que pode estar estabelecido o atraso puberal.

\section{Método}

Foram analisadas 35 pacientes com idades variando de zero a 32,6 anos (média de 11,8 anos), diagnosticadas de 2004 a 2007 no ambulatório do Grupo Interdisciplinar de Estudos da Determinação e Diferenciação do Sexo (GIEDDS) do Hospital de Clínicas da Universidade Estadual de Campinas (Unicamp). Dentre elas, foram incluídas no estudo 29 com mais de dois anos, uma vez que as lactentes costumam ter apresentação clínica diversa das demais, com predominância de sinais como linfedema de mãos e pés, excesso de pele no pescoço e cardiopatias mais graves, sendo outros sinais dismórficos menos evidentes nessa faixa etária. Das 29 pacientes, sete tinham cariótipo 45, X, quatro 45, X/46, XX e as demais apresentavam aberrações estruturais de cromossomos sexuais com ou sem mosaicismo com linhagem 45,X.

Foram obtidos, retrospectivamente, os seguintes dados por meio da análise dos prontuários: antecedentes pessoais 
(doenças cardiovasculares; hipotireoidismo; infecção de trato urinário de repetição e/ou anomalias renais); dados familiares (escolaridade dos pais classificada em $\leq 8$ ou $>8$ anos de estudo, estatura materna classificada em escore Z, recorrência familiar de baixa estatura e número de irmãos classificado em 0-2 e >3); exame físico do paciente (estatura da paciente em escore $Z$ e sinais dismórficos selecionados entre os mais evidentes ao exame clínico e/ou mais característicos da ST, como ptose palpebral, estrabismo, pregas epicânticas, pescoço alado, peito escavado, linfedema residual no dorso dos dedos/artelhos, cubitus valgus e nevos pigmentados).

Os dados das pacientes diagnosticadas com dois a 13 anos, idade a partir da qual se considera atraso puberal no sexo feminino $^{(9,10)}(\mathrm{n}=18)$, foram comparados aos daquelas com idade maior ou igual a 13 anos $(n=11)$, por meio do teste exato de Fisher e do teste $t$ de Student para comparação de médias, com nível de significância de 5\%. Este trabalho foi aprovado pelo Comitê de Ética em Pesquisa da Faculdade de Ciências Médicas da Unicamp.

\section{Resultados}

Não houve diferença significativa entre os dois grupos quanto à estatura da mãe, sendo a média do escore $Z$ entre as 18 mães de pacientes diagnosticadas antes dos 13 anos de $-0,39$ (desvio padrão $-\mathrm{DP}=1,13$ ) e a daquelas diagnosticadas posteriormente $(\mathrm{n}=5)$ de $-0,62(\mathrm{DP}=0,98)(p=0,69)$. Em relação à estatura das próprias pacientes, o déficit de crescimento foi superior nas 18 diagnosticadas antes dos 13 anos (média: -3,29; $\mathrm{DP}=1,51$ ) em relação às outras 11 (média: -2,90; $\mathrm{DP}=1,36)$, mas também não foi estatisticamente significante $(p=0,49)$. Não houve associação entre a época do diagnóstico e a presença dos antecedentes mórbidos considerados individualmente (Tabela 1 ), a escolaridade das mães $(p=0,19)$ e dos pais $(p=0,39)$, a recorrência familiar de baixa estatura $(p=1,00)$ e a presença de sinais dismórficos (considerados tanto individualmente quanto no total) (Tabela 2).

Os dois grupos diferiram, quanto à história de ao menos um antecedente mórbido sugestivo da ST (sendo o diagnóstico mais precoce na presença de ao menos um desses

Tabela 1 - Presença de antecedentes mórbidos entre as pacientes com síndrome de Turner diagnosticadas com idade de dois a 13 anos $e \geq 13$ anos

\begin{tabular}{|c|c|c|c|c|}
\hline Antecedentes mórbidos & Presente & Ausente & Total & Valor de $p$ \\
\hline Doença cardiovascular & & & & 0,26 \\
\hline 2 a 13 anos & $3^{*}$ & 15 & 18 & \\
\hline$\geq 13$ anos & 0 & 11 & 11 & \\
\hline Total & 3 & 26 & 29 & \\
\hline Hipotireoidismo primário & & & & 0,26 \\
\hline 2 a 13 anos & 4 & 14 & 18 & \\
\hline$\geq 13$ anos & 0 & 11 & 11 & \\
\hline Total & 4 & 25 & 29 & \\
\hline ITU de repetição & & & & 0,11 \\
\hline 2 a 13 anos & 7 & 11 & 18 & \\
\hline$\geq 13$ anos & 1 & 10 & 11 & \\
\hline Total & 8 & 21 & 29 & \\
\hline Malformações de vias urinárias & & & & 1,00 \\
\hline 2 a 13 anos & $1^{* *}$ & 17 & 18 & \\
\hline$\geq 13$ anos & 0 & 11 & 11 & \\
\hline Total & 1 & 28 & 29 & \\
\hline Algum dos antecedentes acima & & & & 0,006 \\
\hline 2 a 13 anos & 12 & 6 & 18 & \\
\hline$\geq 13$ anos & 1 & 10 & 11 & \\
\hline Total & 13 & 16 & 29 & \\
\hline
\end{tabular}

* Coartação da aorta e/ou valva aórtica bicúspide; **rim em ferradura.

ITU: infecção do trato urinário. 
Tabela 2 - Comparação dos achados de exame físico entre as pacientes com síndrome de Turner diagnosticadas com idade de dois a 13 anos $e \geq 13$ anos

\begin{tabular}{|c|c|c|c|c|}
\hline Sinais dismórficos & Ausente & Presente & Total & Valor de $p$ \\
\hline Ptose palpebral & & & & 0,37 \\
\hline 2 a 13 anos & 13 & 5 & 18 & \\
\hline$\geq 13$ anos & 9 & 1 & 10 & \\
\hline Total & 22 & 6 & 28 & \\
\hline Estrabismo & & & & NC \\
\hline 2 a 13 anos & 18 & 0 & 18 & \\
\hline$\geq 13$ anos & 10 & 0 & 10 & \\
\hline Total & 28 & 0 & 28 & \\
\hline \multicolumn{5}{|l|}{ Pregas epicânticas } \\
\hline 2 a 13 anos & 16 & 2 & 18 & 1,00 \\
\hline$\geq 13$ anos & 9 & 1 & 10 & \\
\hline Total & 25 & 3 & 28 & \\
\hline Pescoço alado & & & & 0,60 \\
\hline 2 a 13 anos & 16 & 2 & 18 & \\
\hline$\geq 13$ anos & 8 & 2 & 10 & \\
\hline Total & 24 & 4 & 28 & \\
\hline Peito escavado & & & & 0,06 \\
\hline 2 a 13 anos & 12 & 6 & 18 & \\
\hline$\geq 13$ anos & 10 & 0 & 10 & \\
\hline Total & 22 & 6 & 28 & \\
\hline Linfedema residual & & & & 1,00 \\
\hline 2 a 13 anos & 17 & 1 & 18 & \\
\hline$\geq 13$ anos & 10 & 0 & 10 & \\
\hline Total & 27 & 1 & 28 & \\
\hline Cubitus valgus & & & & 1,00 \\
\hline 2 a 13 anos & 4 & 14 & 18 & \\
\hline$\geq 13$ anos & 2 & 8 & 10 & \\
\hline Total & 6 & 22 & 28 & \\
\hline Nevi pigmentados & & & & 0,24 \\
\hline 2 a 13 anos & 7 & 11 & 18 & \\
\hline$\geq 13$ anos & 7 & 3 & 10 & \\
\hline Total & 14 & 14 & 28 & \\
\hline 2 ou mais sinais & & & & 0,19 \\
\hline 2 a 13 anos & 14 & 4 & 18 & \\
\hline$\geq 13$ anos & 9 & 1 & 10 & \\
\hline Total & 23 & 5 & 28 & \\
\hline
\end{tabular}


antecedentes) $(p=0,006)$ (Tabela 1) e ao número de irmãos. Este foi maior quando o diagnóstico foi tardio, sendo que 14 das 18 meninas diagnosticadas antes dos 13 anos tinham de zero a dois irmãos e nove dentre as 11 diagnosticadas após os 13 anos tinham três ou mais irmãos $(p=0,003)$. O maior número de irmãos associou-se, por sua vez, à menor escolaridade da mãe: dentre os 26 casos em que ambos os dados foram obtidos, sete das 14 mães com até dois filhos tinham, no máximo, o ensino fundamental, enquanto que apenas uma das 12 mães com três ou mais filhos havia cursado ensino médio ou superior $(p=0,04)$.

\section{Discussão}

A média de idade ao diagnóstico das pacientes que compõem esta amostra ( 11,8 anos) é mais elevada que a observada atualmente em outros países: 7,7 anos nos Estados Unidos ${ }^{(11)}$ e 6,6 anos na Bélgica ${ }^{(12)}$. Mesmo assim, entre pacientes norteamericanas, constatou-se que o diagnóstico da ST foi feito, em média, 5,2 anos depois que a estatura encontrava-se abaixo do $5^{\circ}$ percentil na curva de crescimento ${ }^{(11)}$.

Na prática diária, em nosso meio, é comum o diagnóstico de pacientes com ST já adolescentes ou mesmo adultas, nas quais a baixa estatura havia sido percebida pela família desde a infância, mas que foram encaminhadas para avaliação somente quando o atraso puberal já era evidente. É possível supor que o atraso no diagnóstico, ou seja, seu estabelecimento apenas após a manifestação de atraso puberal, seja ao menos em parte decorrente de características das próprias pacientes e de suas famílias.

A falta de diferenças significativas em relação à estatura materna não corroborou a hipótese de que a baixa estatura da mãe contribuiu para o atraso no diagnóstico de suas filhas pela suposição, por parte dos médicos e da própria família, de que o déficit de crescimento da criança tivesse origem familiar. O mesmo é sugerido pelo fato de não haver associação entre atraso no diagnóstico e história familiar de baixa estatura.

Por outro lado, chamou a atenção nesta amostra o fato de não haver diferenças significativas em relação ao desvio da estatura das pacientes com menos e mais de 13 anos em relação à média. Uma vez que na ST há queda progressiva da velocidade de crescimento com o passar dos anos ${ }^{(13)}$, era de se esperar que as pacientes diagnosticadas mais tardiamente apresentassem déficit na estatura significativamente maior pelo contrário, esse déficit foi maior naquelas diagnosticadas mais cedo, embora não tenha atingido o nível de significância. Esse resultado permite especular que, nas pacientes com atraso no diagnóstico, a baixa estatura tivesse sido menos acentuada na infância.

Ao se compararem os demais dados das pacientes da amostra com base na idade a partir da qual se configura o atraso puberal, também não houve diferenças significativas em relação à maioria dos dados analisados. No que se refere ao reconhecimento de determinados dismorfismos e anomalias congênitas e adquiridas sugestivas da ST - como pescoço alado, cúbito valgo e múltiplos nevos pigmentados, cardiopatias (coartação da aorta, valva aórtica bicúspide), anomalias renais e de vias urinárias (rim em ferradura, duplicação do sistema coletor) e doença tireoideana autoimune ${ }^{(5)}-$, esses resultados indicam a necessidade de aprimorar a formação médica, particularmente do pediatra, para reconhecer esses e outros sinais associados a essa aberração cromossômica. De fato, várias pacientes, embora apresentassem esses sinais, não foram diagnosticadas precocemente.

Deve-se levar em consideração, porém, o fato de que nenhuma das pacientes incluídas na amostra apresentava mais do que quatro dentre os nove sinais selecionados como mais evidentes ou característicos da ST, indicando que quadros clínicos "típicos", como os descritos em livros-texto, não são observados rotineiramente.

Quando os grupos de pacientes foram comparados em relação à história de ao menos um antecedente mórbido sugestivo da ST, foi possível observar associação significativa com o diagnóstico mais precoce. Tal achado sugere que a associação da baixa estatura à presença de uma das anomalias analisadas torna mais provável reconhecer a necessidade de investigação mais aprofundada, possivelmente por meio do encaminhamento das pacientes a serviços de atenção secundária ou terciária.

As pacientes diagnosticadas com mais de 13 anos diferiram das demais quanto ao número de irmãos (que, diferentemente do que se supunha, foi maior no diagnóstico tardio), e foi possível observar associação significativa entre maior número de irmãos e baixa escolaridade materna, sugerindo a interferência, nesse caso, de fatores socioeconômicos, como baixa renda familiar e pouco acesso a serviços de saúde. Numa situação como essa, é menos provável a procura do serviço médico para investigação da baixa estatura, diferentemente do que ocorre em situações relacionadas a problemas agudos e de urgência.

Conclui-se que, nesta amostra, o diagnóstico precoce da ST esteve relacionado mais à presença de algum dos antecedentes mórbidos relacionados a essa síndrome - o que provavelmente determina encaminhamento a serviços de 
atenção secundária ou terciária - do que à presença de sinais dismórficos. Por outro lado, há indicações de que o déficit de crescimento menos evidente, a dificuldade por parte dos médicos em reconhecer anomalias sugestivas dessa síndrome e determinantes socioeconômicos contribuam para o atraso no diagnóstico.

A redução na idade ao diagnóstico da ST em nosso meio dependerá, portanto, não só da ampliação e melhoria do serviço de atenção básica à saúde, mas também do aprimoramento da formação médica - particularmente do pediatra - para reconhecer o espectro de manifestações clínicas dessa síndrome, incluindo sinais dismórficos,

\section{Referências bibliográficas}

1. Zinn AR, Tonk VS, Chen Z, Flejter WL, Gardner HA, Guerra R et al. Evidence for a Turner syndrome locus or loci at Xp11.2-p22.1. Am J Hum Genet 1998;63:1757-66.

2. Nielsen J, Wohlert M. Chromosome abnormalities found among 34,910 newborn children: results from a 13-year incidence study in Arhus, Denmark. Hum Genet 1991;87:81-3.

3. Viguetti NL, Maciel-Guerra AT. Short stature and Turner syndrome: an association more frequent than expected. J Pediatr (Rio J) 1994;70:172-4.

4. Sybert VP, McCauley E. Medical progress: Turner's syndrome. N Eng J Med 2004;351:1227-33.

5. Bondy CA. Turner Syndrome Study Group. Care of girls and women with Turner syndrome: a guideline of the Turner Syndrome Study Group. J Clin Endocrinol Metab 2007;92:10-25.

6. Guedes AD, Verreschi IT; Grupo de Discussão em Síndrome de Turner. Síndrome de Turner: diagnóstico e tratamento. Projeto diretrizes 2006. São Paulo: Associação Médica Brasileira; Conselho Federal de Medicina; 2006. anomalias viscerais congênitas e condições adquiridas. É fundamental, ainda, ampliar os serviços de genética no país, tornando possível a análise cromossômica rotineira de meninas com baixa estatura de origem indefinida, como sugerido por vários autores em consensos nacionais e internacionais ${ }^{(5,6,11)}$.

\section{Agradecimentos}

Ao Laboratório de Citogenética Humana do Departamento de Genética Médica da Faculdade de Ciências Médicas da Unicamp.

7. Suzigan LZC, Silva RBP, Maciel-Guerra AT. Aspectos psicossociais da síndrome de Turner. Arq Bras Endocrinol Metab 2005;49:157-64.

8. Carvalho AB, Guerra-Junior G, Baptista MT, Marques-de-Faria AP, LemosMarini SH, Maciel-Guerra AT. Turner syndrome: a pediatric diagnosis frequently made by non-pediatricians. J Pediatr (Rio J) 2010;86:121-5.

9. Lee PA. Normal ages of pubertal events among American males and females. J Adolesc Health Care 1980;1:26-9.

10. Spinola-Castro AM. Retardo puberal. In: Monte O, Longui CA, Calliari LE, Kochi C, editores. Endocrinologia para o pediatra. $3^{a}$ ed. São Paulo: Atheneu; 2006. p. 167-74.

11. Sävendahl L, Davenport ML. Delayed diagnoses of Turner's syndrome: proposed guidelines for change. J Pediatr 2000;137:455-9.

12. Massa G, Verlinde F, De Schepper J, Thomas M, Bourguignon JP, Craen M et al. Trends in age at diagnosis of Turner syndrome. Arch Dis Child 2005;90:267-8.

13. Lyon AJ, Preece MA, Grant DB. Growth curve for girls with Turner syndrome. Arch Dis Child 1985;60:932-5. 